\title{
BMJ Open Family planning and pregnancy issues for women with systemic inflammatory diseases: patient and physician perspectives
}

\author{
Eliza Chakravarty, ${ }^{1}$ Megan E B Clowse, ${ }^{2}$ Daphnee S Pushparajah, ${ }^{3}$ \\ Sarah Mertens, ${ }^{3}$ Caroline Gordon ${ }^{4}$
}

To cite: Chakravarty E, Clowse MEB,

Pushparajah DS, et al. Family planning and pregnancy issues for women with systemic inflammatory diseases: patient and physician perspectives. BMJ Open 2014;4:e004081. doi:10.1136/bmjopen-2013004081

- Prepublication history and additional material for this paper is available online. To view these files please visit the journal online (http://dx.doi.org/10.1136/ bmjopen-2013-004081).

Received 25 September 2013 Revised 18 December 2013 Accepted 14 January 2014

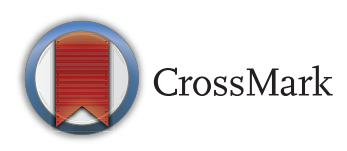

${ }^{1}$ Oklahoma Medical Research Foundation, Oklahoma City, Oklahoma, USA ${ }^{2}$ Duke University Medical Center, Durham, North Carolina, USA

${ }^{3}$ UCB Pharma, Brussels, Belgium

${ }^{4}$ School of Immunity and Infection, College of Medical and Dental Sciences, University of Birmingham, Birmingham, UK

Correspondence to Dr Eliza Chakravarty; Eliza-Chakravarty@omrf.org

\section{ABSTRACT}

Objectives: To identify family planning and pregnancy (FPP) issues for female patients of childbearing age living with a chronic inflammatory disease and to assess whether current clinical practice routinely provides adequate support to alleviate these concerns.

Setting: Multinational survey and an analysis of online patient activity.

Participants: Premenopausal women (aged 20-45 years; $\mathrm{N}=969$ ) were surveyed in the USA, the UK, Germany, France, Italy and Spain. Rheumatologists were surveyed in Germany $(\mathrm{N}=50)$, France $(\mathrm{N}=50)$, Italy $(\mathrm{N}=50)$ and the USA $(\mathrm{N}=100)$, and gastroenterologists were also surveyed in the USA ( $N=100)$.

Primary and secondary outcome measures: Two online surveys were undertaken to identify FPP issues for physicians and patients. The surveys examined the frequency of dialogue on these topics between physicians and patients, alongside assessment of patient satisfaction regarding these conversations. Online analysis identified key themes for patient discussion outside their doctors' office/clinic/surgery.

Results: $32-56 \%$ of physicians spontaneously reported having talked about FPP with their female patients of childbearing age. When prompted, the majority of rheumatologists (74-92\%) and gastroenterologists $(74 \%)$ reported having discussed conception/pregnancy with female patients; however, less than half reported consulting their patient's treating general practitioner/gynaecologist about these topics. The majority of patients reported their FPP-related concerns are not adequately addressed/ settled during their medical appointments. Furthermore, only $30-40 \%$ of patients considered advice/information to be consistent across multiple healthcare professionals. Key online FPP-related patient discussions included disease state, adverse effects, treatment, switch behaviour and wash-out requirements.

Conclusions: Female patients who live with chronic inflammatory disease have important FPP concerns. The majority of patients, however, do not feel that their FPP concerns are adequately addressed in current clinical practice and report that they receive inconsistent advice from the various healthcare professionals who manage different aspects of their care. There is a clear need for

\section{Strengths and limitations of this study}

Strength: Investigation of patient and physician perspectives.

- Strength: Involvement of specialties from different areas of interest.

- Strength: Cross-cultural investigation.

- Weakness: Absence of formal survey validation.

- Weakness: Reliance on patient self-reporting.

provision of up-to-date and consistent information/ support to female patients.

\section{INTRODUCTION}

Autoimmune and inflammatory diseases often affect women of reproductive age. Although rheumatoid arthritis (RA) becomes more common as patients age, ${ }^{1}$ women are increasingly choosing to start their family later in life and treatments to control RA are now often started at a younger age. Moreover, even though only a minority proportion of patients with RA are women of childbearing age, ${ }^{2}$ the high prevalence of the disease means that it does impact a significant number of young women. Many other inflammatory diseases, including ankylosing spondylitis, ${ }^{34}$ Crohn's disease (CD) ${ }^{56}$ inflammatory bowel disease (IBD) ${ }^{7}$ and systemic lupus erythematosus (SLE), ${ }^{8}$ also affect a younger population and thus have a direct effect on women of childbearing age.

Pregnancy and child rearing are important facets of life for most women. Now that improved therapies for inflammatory diseases have enabled better physical function and quality of life, many women who previously would have felt too ill to consider child bearing are now better able to fulfil desires for family life. Family planning and 
pregnancy (FPP) are also important issues for this patient population as these disease states have been linked to an increased risk of adverse pregnancy outcomes, ${ }^{9-11}$ including increased risk of preterm birth, ${ }^{12}$ difficulty carrying to full term ${ }^{13}$ and a possible reduction in fertility, ${ }^{14}{ }^{15}$ although there is conflicting evidence regarding the impact of inflammatory diseases on fertility. ${ }^{16} 17$ Owing to the impact on pregnancy outcomes, expert advice is to achieve and maintain stable low disease activity prior to conception and throughout pregnancy. ${ }^{18-20}$ Some anti-inflammatory treatment options can be potentially hazardous for pregnant women to take as drugs can pass across the placenta and may affect the fetus. ${ }^{21}$ Methotrexate, a common RA treatment, can be damaging to fetal development (at least at high doses) $)^{22} 23$ while other disease-modifying antirheumatic drugs such as leflunomide have been shown to cause malformations in animal studies. ${ }^{24}$

However, use of medications during pregnancy is not always contraindicated and an individual risk-benefit discussion should be undertaken between the patient and expert physician to provide the best management of the disease and the pregnancy. ${ }^{25}$ Inadequate dissemination of appropriate advice describing which drugs may be continued can lead to women unnecessarily forgoing potentially helpful medications and thus suffering throughout pregnancy, with the increased risk of further complications to both mother and child due to the effects of active disease. ${ }^{26}$ Furthermore, patients are increasingly seeking additional information on the internet, ${ }^{27}$ while a recent analysis of information regarding medication safety on active internet sites has noted an inadequate evidence base for the advice often provided and inconsistent guidance. ${ }^{28}$ It is clear that communication of reliable and consistent information is required to enable the correct treatment of these women before and during pregnancy, and while breastfeeding. Education and information should be shared by healthcare professionals dealing with these diseases, medications and situations on a daily basis. Accurate, consistent information must be communicated to women considering or entering pregnancy in order to support patients through this delicate journey.

Two surveys were undertaken to investigate some of these issues, one for physicians and another for female patients, to identify the key concerns of both groups related to the topics of FPP in inflammatory disease. The surveys were designed to gauge whether there is a gap in the communication between what healthcare professionals provide in terms of information/support and what patients feel that they receive. The patient survey also investigated where patients go to seek additional information. Two key objectives of the study were to examine the proportion of physicians who discuss FPP issues with their female patients of childbearing age and the proportion of patients who have discussed these topics, in the context of their condition, with their healthcare professional. The survey also assessed the proportion of patients who feel that their concerns on this topic have been satisfactorily addressed by these discussions. We aim to communicate the insights gained from this investigation to clinicians treating women of childbearing age, who live with chronic inflammatory conditions, in order to provide advice on how best to support these patients.

\section{METHODS}

This study complied with the ICC/ESOMAR, EphMRA, ABPI, MRS and BHBIA market research codes of conduct, ensuring the anonymity and confidentiality of all participants. Respondents were paid a nominal amount, calculated using fair market value guidance, to compensate for the time and effort of completing the survey.

\section{Physician survey}

The online physician questionnaire was delivered in two phases, the first phase (baseline) was distributed in July 2012 (Europe) and September 2012 (USA), and the second phase was distributed in November 2012 in Europe and the USA. The survey was delivered in the local language and translations were checked for consistency against the original in English by native speakers with fluency in both languages (the complete Physician Questionnaire can be found in online supplementary appendix 1). The questionnaire was designed to elicit spontaneous (open-ended question) and prompted (closed question in which FPP topics were an option among other answering categories) responses. Participants were recruited from the WorldOne Physician Panel. Rheumatologists were surveyed in four countries: Germany $(\mathrm{N}=50)$, France $(\mathrm{N}=50)$, Italy $(\mathrm{N}=50)$ and the USA $(\mathrm{N}=100)$. Gastroenterologists were surveyed in the USA $(\mathrm{N}=100)$. Responses were compared between phases using two-sided z-tests with significance level 0.05 , which corresponds to a CI of $95 \%$.

Participating physicians had to meet the following criteria: $3-30$ years of experience, $\geq 50 \%$ patient-facing time, had not participated in medical research within their specialist area in the past month, were not currently a clinical investigator for a pharmaceutical manufacturer and were not currently active in medical research or advertising (to avoid inclusion of respondents motivated only by receipt of payment). Physicians with rheumatology as their primary specialty had to meet the following additional criteria: treat $\geq 20 \mathrm{RA}$ patients/month and have $\geq 10$ patients on biologics/ month. Physicians with gastroenterology as their primary specialty had to meet the following additional criteria: treat $\geq 8 \mathrm{CD}$ patients per/month and have $\geq 2$ patients on biologics/month. These selection criteria were applied to ensure participating physicians had sufficient experience treating patients with systemic inflammatory disease in order to provide informative results, while 
also seeking to include physicians with a range of experience in order to investigate routine clinical practice.

\section{Patient survey}

The online patient questionnaire was designed according to standard market research survey methodology, including scaling questions and avoiding skewed questions. The questionnaire was delivered in the local language and translations were checked for consistency against the original in English by native speakers with fluency in both languages (the complete Patient Questionnaire can be found in online supplementary appendix 2). The questionnaire was targeted at premenopausal women (age was self-declared and the survey excluded women under 20 and over 45 years of age; see Question 3 Patient Survey). A professional recruitment agency was used to recruit patients from the GlobalTestMarket and MySurvey online survey panels. Respondents were screened according to age, gender and disease (all self-reported) and those who were $<20$ or $>45$ years old, male, or not suffering from RA, CD or lupus were excluded. Recruited patients were then sent a link to an online survey, which was followed up by a short telephone interview at a prearranged time.

The patient questionnaire was delivered in two phases and was distributed in six countries (the USA, the UK, Germany, France, Italy and Spain). The first phase (baseline) consisted of 16 questions and was disseminated to patients between 17 July 2012 and 15 August 2012. There were 1069 respondents to the first phase which covered patients with RA, SLE, CD and ulcerative colitis (UC). The second phase consisted of the original 16 questions from the first phase plus an additional 8 questions included in order to avoid so-called false positives (ie, covered topics included in other questions to test consistency of response) and to provide greater insight into patients' experience in terms of medical care and needs. The second phase elicited 969 responses and was distributed between 13 October 2012 and 16 November 2012 to patients with axial spondyloarthritis and psoriatic arthritis, in addition to patients with RA, SLE, CD and UC. All responses were anonymous. The patients who were invited to participate in the two survey phases were not identical, although there may have been some overlap (responses were anonymous therefore the extent of overlap could not be determined).

\section{Netnography research}

Online discussions (English language only) relating to FPP issues were investigated using two approaches: social media monitoring and search engine landscape/content analysis (SELA).

Social media monitoring was undertaken by identifying categories of interest (defined as: 'ulcerative colitis', 'systemic lupus erythematosus', 'rheumatoid arthritis', 'colitis ulcerosa', 'lupus', 'regional enteritis' and 'Crohn's disease') and keywords of interest (defined as: 'pregnancy', 'miscarriage', 'birth control', 'miscarriages', 'fertile', 'family planning', 'gestation', 'pregnant', 'fertility', 'pregnancy disease evolution', 'disease transfer to baby', 'breastfeeding', 'breast feeding', 'conception', 'conceived' and 'placental transfer'), and then combining keywords together with the categories of interest to ensure coverage of all topics of interest.

SELA methodology involved identification of keywords (including generic keywords/keyword phrases and specific keywords/keyword phrases), assessment of keyword search volume and ranking of individual sites in search engine results. The 'click through rate' was combined with the 'monthly search volume' to estimate the 'share of attention index'. The final keyword pool included the categories 'colitis ulcerosa', 'ulcerative colitis', 'Crohn's disease', 'lupus', 'rheumatoid arthritis', 'regional enteritis' and 'systemic lupus erythematosus', and the following FPP-related keywords 'gestation', 'birth control', 'pregnancy', 'fertility', 'miscarriage' and 'family planning'.

\section{RESULTS}

\section{Physician survey}

Many rheumatologists and gastroenterologists spontaneously reported having discussed FPP-related topics with their female patients of childbearing age; reports were consistent across all countries studied (figure 1A). Interestingly, the number of US gastroenterologists who discussed these topics with their female patients increased significantly between the first and second phases of the survey (figure 1A). This was particularly true for fertility, pregnancy and/or family planning issues specifically, in which the percentage of US gastroenterologists discussing these topics increased from $67 \%$ to $79 \%$ between the two phases (significant difference, 95\% confidence level; $\mathrm{N}=100$ for both survey phases); however, there was no difference in European practice, with levels remaining at $60 \%$.

When prompted, the majority of rheumatologists (74$92 \%)$ and gastroenterologists (74\%) reported having discussed conception/pregnancy with female patients of childbearing age. However, less than half of these physicians discussed FPP-related issues with their patient's treating general practitioner (GP; ie, primary care physician) or gynaecologist (figure 1B,C; survey phase 2 data shown; see Questions 20 and 21 Physician Survey online supplementary appendix 1).

When seeking additional information regarding FPP, community rheumatologists and gastroenterologists reported currently relying on presentations and educational events at congresses, other healthcare professionals and key opinion leaders as their preferred sources of information.

\section{Patient survey and netnography analysis}

Patients reported that the frequency of FPP-related conversations during their medical appointments was lower than their discussions about emotional well-being and 
employment (figure 2A), although most female patients of childbearing age reported discussing these issues at some point during their care pathway (figure 2B).

Patients currently on medication for the treatment of their condition reported being more concerned about FPP issues than those not receiving medication, with $63 \%$ of those on medication reporting that pregnancy was a concern compared with only $32 \%$ of patients not receiving medication. In addition, the female age group with most concerns related to FPP was between the ages of 30 and 34 (figure 2C; survey phase 2 data shown, similar trend observed in phase 1). Concern was high between the ages of 25 and 39 but decreased in patients aged between 40 and 45 .

Patients were asked when they would prefer to discuss the topics of FPP. The survey revealed patients prefer to discuss these issues in the context of their disease and treatment whenever a decision is made that could have an impact on their family planning or ability to become pregnant (figure 3A). Approximately a quarter feel that

\section{A) Proportion of physicians who report having discussed family planning topics with female patients of child-bearing age (last 3 patients were considered)}

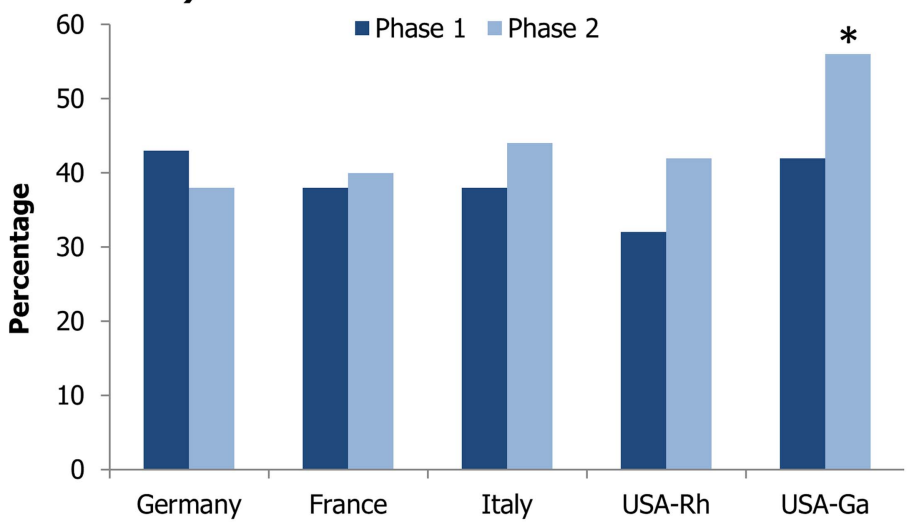

B) To what extent did you discuss these questions, issues...with the patient's GP? [see Question 20 of the Physician Survey, Appendix 1]

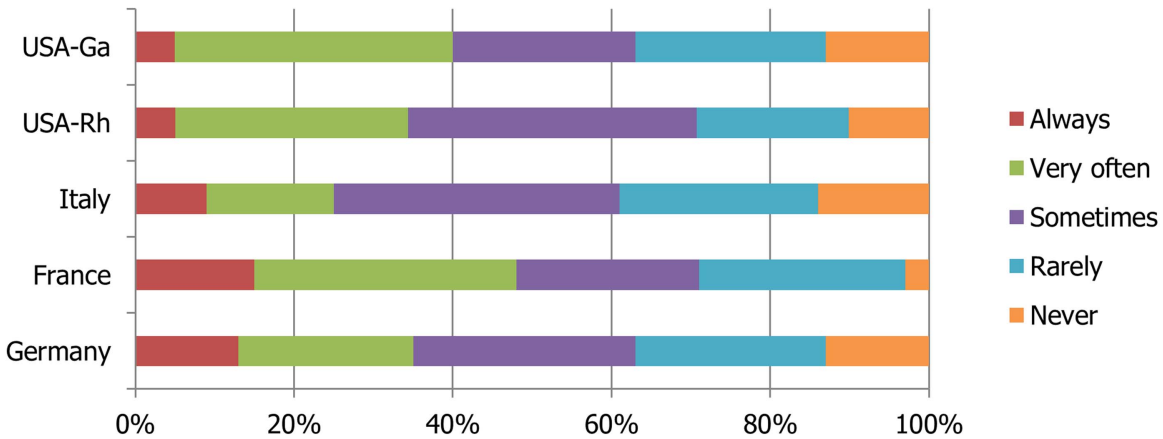

C) To what extent did you discuss these questions, issues...with the patient's gynaecologist? [see Question 21 of the Physician Survey, Appendix 1]

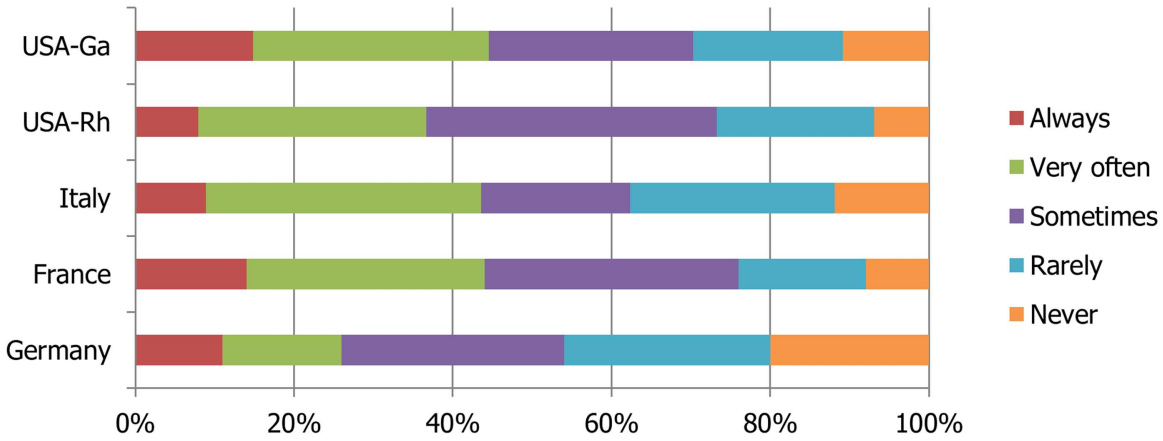

Figure 1 Frequency of physician-initiated discussions regarding family planning and pregnancy issues with female patients and their general practitioners or gynaecologists (survey phase 2 data shown in B and C). Ga, gastroenterologist; Rh, rheumatologist. ${ }^{*} \mathrm{p}<0.05$ compared to phase 1 (two-sided z-test with significance level 0.05 ). 
A) Topics discussed during any of the last three medical appointments

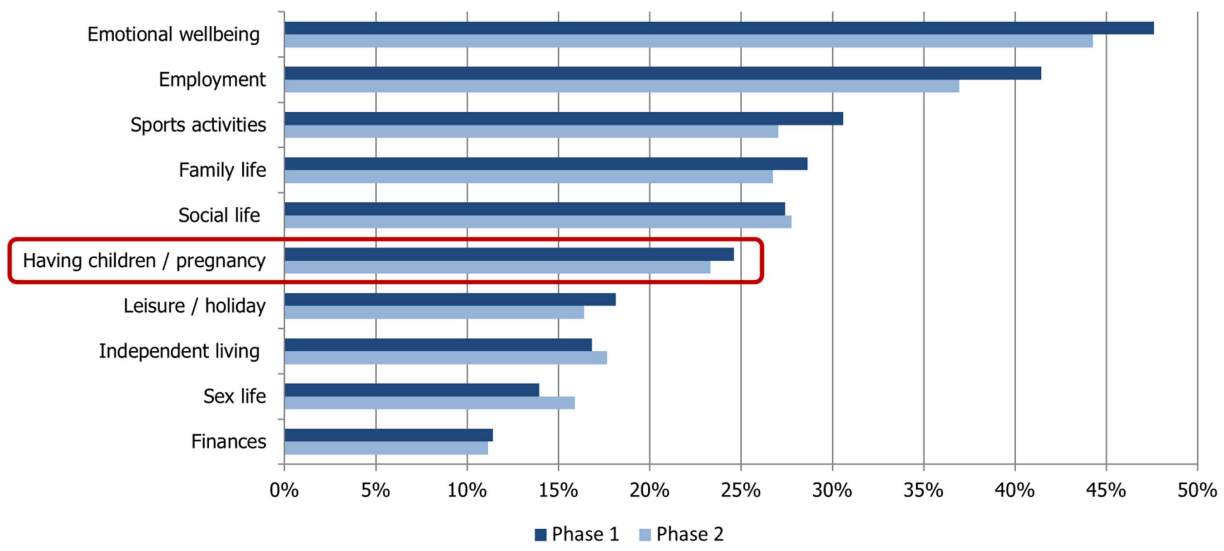

B) Have family planning concerns ever been discussed?

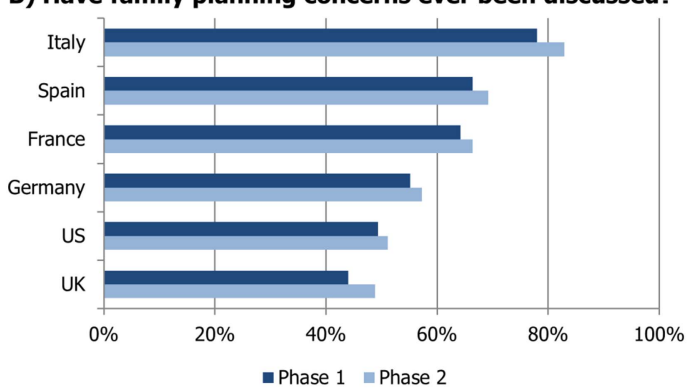

C) Are family planning issues of concern?

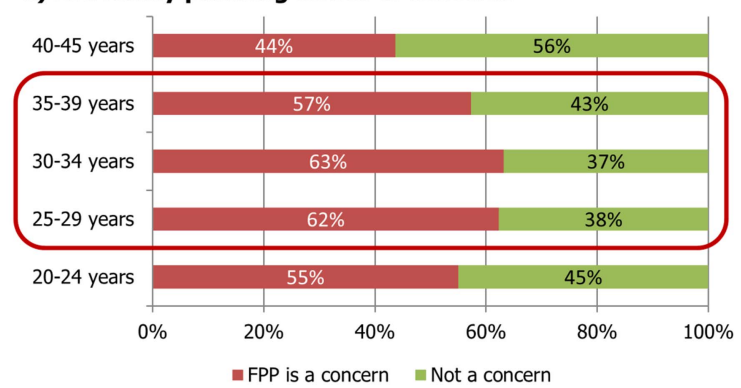

Figure 2 Patient-reported (patient survey) topics of discussion with specialist physicians, the frequency of patient-initiated family planning and pregnancy (FPP) discussion and the importance of FPP issues stratified by patient age (data from survey phase 2 shown in $\mathrm{C}$ ).

one conversation with their healthcare professional on this topic is enough, although some would like to discuss these topics at every visit. Furthermore, approximately a third of patients prefer to initiate conversations on this topic themselves or to obtain information from their healthcare professional when appropriate to their individual situation.

Patients gave a range of reasons for not discussing FPP concerns with their healthcare provider, with the most common reason being that they forgot to mention it (figure 3B). Other important reasons identified as barriers for discussing FPP was the impression that the healthcare professional either did not have time for discussions or that they were not the correct physician to provide advice on these topics. Importantly, some patients also reported that they choose to not discuss FPP issues as they were reluctant to change medication. Very few patients felt that their healthcare professional was reluctant to discuss these topics. Over a quarter of respondents indicated that they had 'other' reasons for not discussing these issues with their doctor (figure 3B). A higher proportion (39\%) of patients aged 40-45 years recorded 'other' reasons for not discussing FPP issues with their doctor, compared with younger patients ('other' was recorded by $21.7 \%$ of $20-24,18.3 \%$ of 25-29, $18.7 \%$ of 30-34 and 25.6\% 35-39-year-olds).

Patients report a preference for seeking information regarding FPP from their gynaecologist (figure 4A), whereas disease specialists (eg, rheumatologist or gastroenterologist) are their key contact point for management of their chronic condition (figure 4B). GPs/ primary care physicians were also identified by patients as central to their discussions of both FPP and their chronic condition (figure 4A-B). It should be noted, however, that patient reports of a preference for one specialist over another regarding FPP discussions could be expected to vary by country given differences in treatment practices. For example, in the USA patients may commonly visit a gynaecologist/obstetrician for all pregnancy care, whereas in the UK the patient's GP and local midwife may oversee most of their pregnancy planning and management. In addition, when responding to the survey some, but not all, patients may have regarded the terms 'obstetrician' and 'gynaecologist' as interchangeable based on their own experience.

Other than their doctors' office/clinic/surgery, patients reported researching and discussing information regarding FPP through multiple channels (figure 4C): predominantly on disease-related websites and with family and friends. In addition, patients reported that specific condition-related forums/patient organisations did not play a prominent part in their search for online information on these topics. A number of key themes, such as discussions around disease state, adverse effects, treatment, switch behaviour and wash-out requirements, emerged after following online patient activity. Other 
A) How often would you like the topic of family planning to be brought up during your medical appointments?

When taking decisions that could have an impact on my family planning

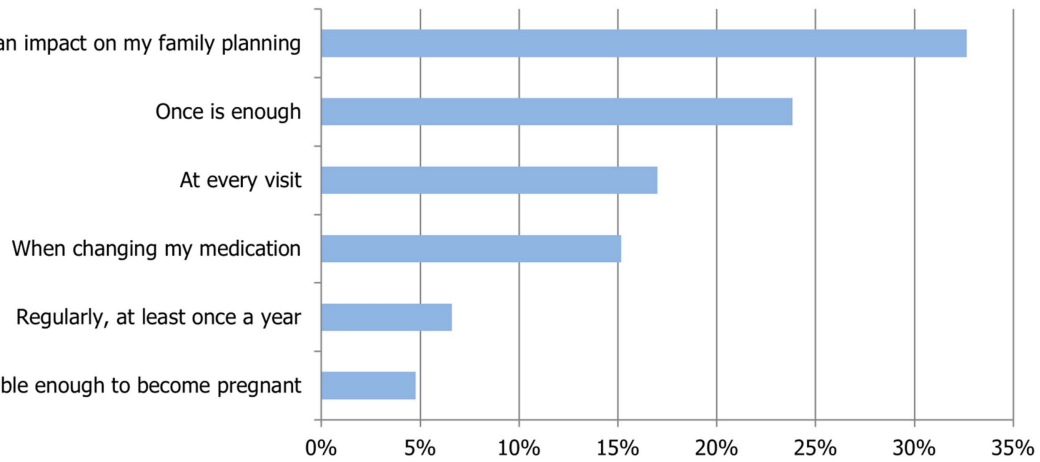

Every time when my (CONDITION) is stable enough to become pregnant

B) Issues that prevented a discussion

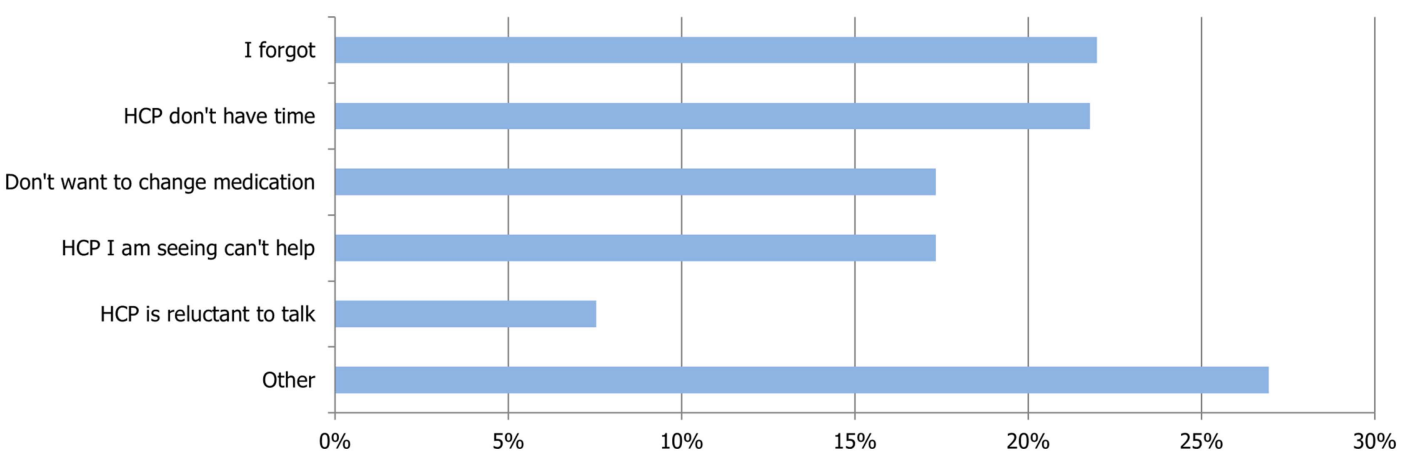

Figure 3 Patient preference for frequency of discussions relating to family planning and pregnancy issues and identification of issues that prevented discussion (data from patient survey phase 2 shown).

themes noted were patient emotions and feelings, interactions with healthcare professionals, how best to identify the correct healthcare professional for their treatment, concerns about inconsistent advice, infertility, sexuality and conception, disease carry-over to the baby, placental transfer of treatments, and breastfeeding.

Importantly, approximately $30-55 \%$ of female patients reported that their concerns relating to FPP are not adequately addressed or settled during their medical appointments (data from patient survey phase 2). Some variability in this response was observed across countries, with $30 \%$ of patients from the USA, 34\% from Italy, 35\% from Spain, 39\% from the UK, $43 \%$ from France and $54 \%$ from Germany reporting that their concerns were not settled (data from patient survey phase 2). Patients also reported that consistency of advice and information given by multiple healthcare professionals, including nurses, was low with only about $30-40 \%$ of patients reporting consistent advice. Again, responses to this question varied across countries with $33 \%$ of patients from Spain and the UK reporting consistent advice, $36 \%$ from Germany and Italy, $40 \%$ from the USA and $41 \%$ from France (data from patient survey phase 2). Inconsistent advice was reported by about $30-50 \%$ of patients overall: $27 \%$ from Italy, 32\% from Spain, 38\% from France, $41 \%$ from the USA, $44 \%$ from Germany and $49 \%$ from the UK (remaining patients selected a neutral response to this question; data from patient survey phase 2).

\section{DISCUSSION}

The investigation described here was carried out to illuminate some of the issues surrounding FPP for female patients of childbearing age who live with chronic inflammatory diseases. Recently it was reported that almost half of female patients with IBD feel their disease and/or treatment influences their decisions about FPP but despite this about two-thirds had not discussed these issues with their doctor. ${ }^{29}$ The results from the current study confirmed that FPP are considered important issues by this group of female patients and that there are key gaps in communication which result in inconsistent advice and subsequent patient concern/confusion. Importantly, the majority of female patients of childbearing age reported that current clinical practice does not adequately address their concerns related to FPP in inflammatory disease.

Some clinical recommendations for the management of inflammatory disease during pregnancy have been published $^{18-20} 2530$ and advise that clinical remission/ stable low disease activity be achieved prior to conception and maintained throughout pregnancy using appropriate therapy as needed. However, in the current study few patients reported discussing FPP at the point their condition was stable enough to become pregnant, suggesting a gap in necessary communication from physicians to patients regarding the need to control disease activity prior to conception, the impact of disease activity 
A) What healthcare provider do you primarily visit for family planning issues?

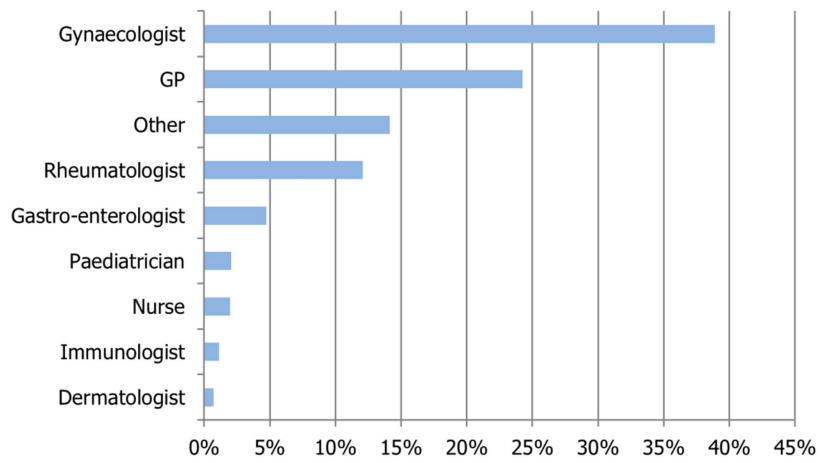

B) What healthcare provider do you primarily visit for your condition?

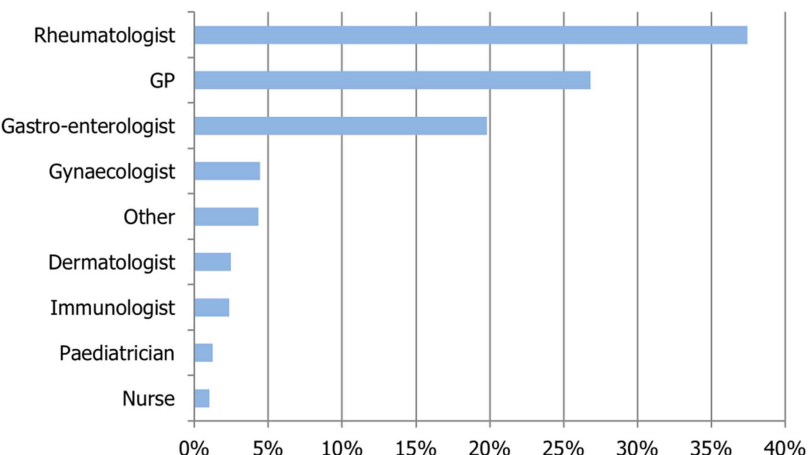

C) Preferred sources of information relating to condition and family planning issues

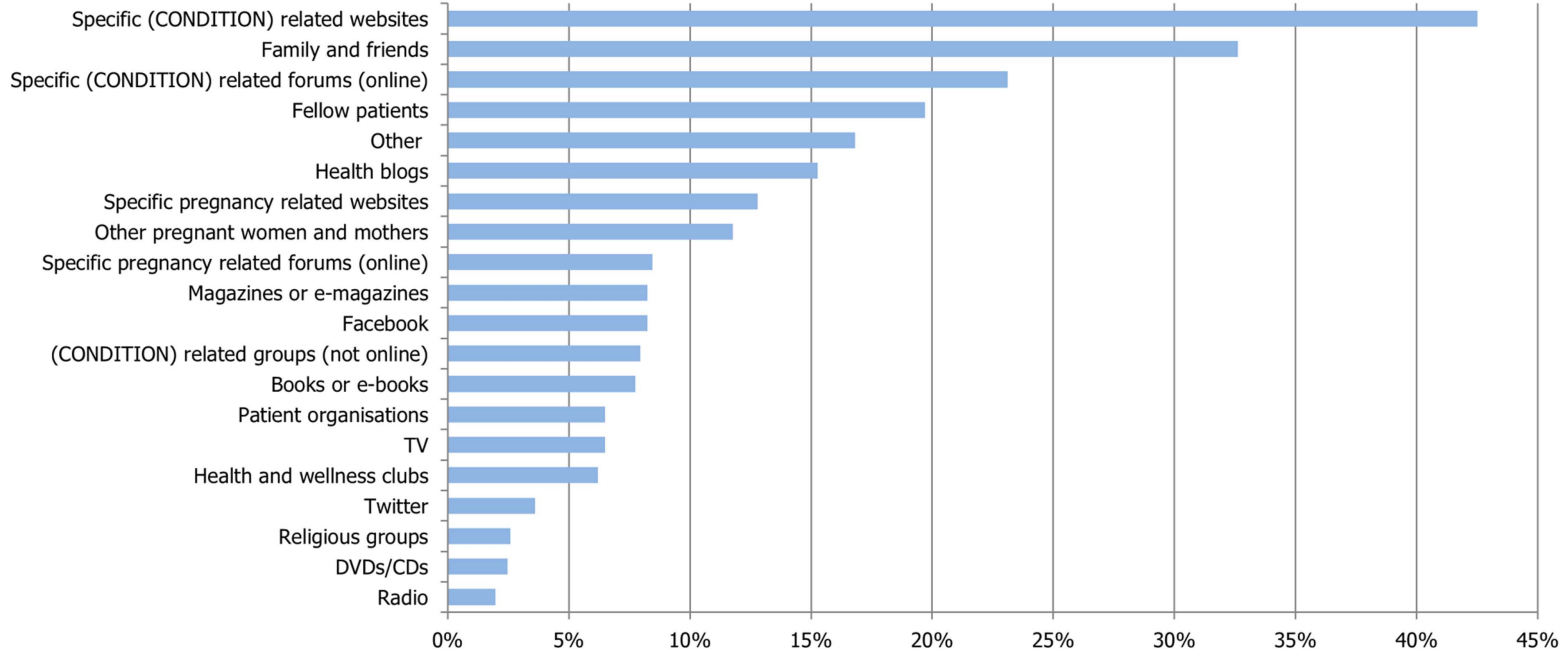

Figure 4 Patient preference for choice of healthcare provider for discussions of their condition and family planning and pregnancy (FPP) issues, and their preferred source of additional information (outside doctors' office/clinic/surgery) relating to their condition and FPP issues (data from patient survey phase 2 shown). GP, general practitioner.

on pregnancy outcomes and the need to adjust medications during pregnancy. Indeed, a general lack of patient knowledge regarding continued use of medication during pregnancy was highlighted recently by a survey of female patients with IBD which revealed a widespread, but inaccurate, belief that all medications needed to be stopped during pregnancy. ${ }^{29}$ Respondents to the current patient survey also reported that inconsistencies in advice regarding the use of anti-inflammatory and immunosuppressive medications during pregnancy are common. Together, these results suggest a need for continuing education of all specialists involved in the care of women with inflammatory disease in order to ensure women understand the implications of their condition and treatment on FPP. Congress presentations and associated education events were identified by physicians, specifically rheumatologists and gastroenterologists, as their currently preferred source of information and continuing education-which may also be applicable to other specialists. As such, these events should be actively targeted to maximise and improve continued education. This could, for example, include hosting discussion forums between specialists involved in different aspects of care for these patients at international and national congresses. Timely discussion of FPP issues is also an important consideration given that a high percentage of pregnancies are unplanned. ${ }^{31}$

GPs/primary care physicians and gynaecologists were identified by patients as frequently central to their discussions on FPP issues, although research has previously shown that $41 \%$ of GPs do not initiate discussion of FPP with female patients affected by IBD. ${ }^{29}$ Importantly a gap in communication was identified between these physicians and the specialists who treat chronic inflammatory diseases. As such, improved cross-specialty communication should be strongly encouraged, particularly for discussions regarding planning and treatment guidelines. This could involve establishment of cross-specialty teams within hospitals/centres of excellence to co-ordinate care of pregnant women living with systemic inflammatory disease. A recent survey of GPs in Ireland did show that the majority of GPs report seeking additional advice on FPP issues, in relation to their female patients with IBD, from tertiary specialists ${ }^{29}$ so improved 
education for all specialties, and fostering communication should assist dissemination of information and consistent advice for patients. Furthermore, all of those involved in the care of female patients of childbearing age who live with chronic inflammatory disease, not only the immunological disease specialists, should be exposed to continued education on this topic. Another recent survey of rheumatologists and obstetricians showed that there is variability in advice given to patients concerning the use of specific medication during pregnancy. ${ }^{32}$ This highlights a need for consistency among clinicians to achieve quality patient care. Development of cross-specialty international guidelines/consensus papers by relevant expert physicians may help bridge these gaps in communication. Furthermore, publication of evidence-based discussions of the risks and benefits of medication use and disease activity control during pregnancy in women with inflammatory diseases, by relevant medical societies, could provide an easily accessible resource for physicians and patients alike. These could be in the form of 'white papers' and accompanying patient information pieces, published in the scientific literature and online, to provide consistent education and advice for cross-specialty physicians and their patients.

In order to improve the dissemination of information regarding FPP to patients, it is important to identify when patients prefer to receive such information and also why this information may not be adequately communicated. Patient preference regarding timing of discussions about FPP varied. This variability may be due to the personal nature of this topic, differences in retention of information and differences in healthcare services/societal norms across different countries. Patients did, however, generally report that they wished to discuss FPP-related issues with their specialist physician (ie, rheumatologist or gastroenterologist) every time a decision was made that could impact on their FPP. As such, healthcare professionals should routinely consider any issues that affect fertility or pregnancy and offer to have a conversation with their patient regarding these issues at every clinic visit, unless it is known not to be relevant. It may also be useful to clarify patient expectations for support and advice during these discussions. Patients on medication were more concerned about FPP issues than those not receiving medication, as were female patients between the ages of approximately 25 and 40 . The high level of concern in both of these groups highlights these patients as key populations requiring additional consideration, although it could be argued that all women of childbearing age should be targeted for such communications.

The most common reason patients offered to explain why they did not raise FPP with their healthcare provider was that they forgot to mention it during their consultation. Many also stated they avoided such discussion as they did not want to change their medication, implying that a fear of destabilising their disease could influence the preparedness of patients to discuss FPP topics. Other key reasons identified were the impression that their physician did not have time for the discussion or that they felt their treating physician was not the correct physician to consult on these topics. Consequently, physicians should consider periodically raising the issue themselves, particularly when treatment decisions or disease activity could impact FPP plans. Notably over a quarter of respondents indicated that they had 'other' reasons for not discussing these issues with their doctor. It is possible that this high response was due to patient demographics (eg, age, social background or current use of contraception) and patients considering their family already complete. Indeed, a higher proportion of older patients recorded 'other' reasons for not discussing FPP issues with their doctor compared with younger patients. Patient emotions was an important theme identified for online discussions, perhaps particularly relevant for those with a history of miscarriage/stillbirth or infertility problems, and it should also be considered that such associations could be a factor underlying why some patients reported that they did not discuss FPP with their physician for 'other' reasons.

Of key importance, many female patients of childbearing age reported that they did not feel that their concerns relating to FPP in the context of their disease were adequately addressed during their medical appointments. There was some variability in patient response across countries to this question, suggesting that cultural differences and differences in healthcare system structure may contribute to the variability in patient satisfaction. However, it is clear that there can be a definite improvement in the response to these issues and all healthcare professionals should consider how they could increase such communication and support to their patients. This could include provision of better patient educational material and advice on reliable and up-to-date websites containing FPP-related information. Indeed, patients reported that they frequently sought information online, although specific condition-related forums/patient organisations did not appear to be common sources of this information. Furthermore, the online landscape was fragmented by disease area, with no specific resource (beyond 'Motherisk'; http://www. motherisk.org) that provides FPP guidance on common autoimmune conditions and medication use affecting women in the reproductive age group. As such, development of a single site with consistent up-to-date guidance covering topics identified by patients as key interests would probably be of great value. Alternatively, patient and/or physician organisations could incorporate more detailed information and advice on their websites to offer improved support to concerned patients and the various physicians involved in their care during pregnancy. Another key improvement would be increased dialogue, by telephone, letters or email, between the varied physicians involved in an individual patient's care in order to provide coordinated advice and support. Together these measures would aim to improve patient support and satisfaction. 
It should be noted that this study does have some limitations. Two important limitations of the patient survey were an absence of formal survey validation and the reliance on self-reporting of diagnosis by patients. It should also be considered that patients who are willing and motivated to complete a questionnaire may differ significantly from the overall patient population. Furthermore, even though the surveys were translated into local languages, differences in terminology and healthcare system structure exist across countries which could impact pooling of results; for example, the terms obstetrician and gynaecologist could be understood as being interchangeable in some countries but distinctly different specialties in others, meaning patient interpretation of the survey could vary. The physician survey may also suffer related issues. In addition, the physician survey was only delivered to gastroenterologists in the USA and did not investigate the activities of these physicians in other countries. The range of experience among physicians was also broad which, although providing good coverage of routine clinical practice, could be complicated by differences in attitudes between physicians of different generations. A limitation of the current study is an inability to tease out any potential influence these factors may have on variation in the results. The netnography research may have been limited by use of keywords common to one country/region but different in others (eg, 'fertility' vs 'infertility') which could have restricted comprehensive analysis of the online landscape and patient discussions. Finally, this study did not investigate the related concerns of men suffering from inflammatory disease who may be using chronic medication and also considering a family; indeed this population is often understudied and may need improved support.

In summary, FPP are extremely important issues for female patients of childbearing age who live with chronic inflammatory disease. A summary of key considerations, highlighted by the results from the current study, for all physicians involved in the treatment of this often neglected group of patients is presented in figure 5. It is clear that female patients of childbearing age do wish to discuss these issues with their physicians, although expectations regarding frequency of discussion and their preferred physician for advice vary considerably. Currently it appears that more can be done to provide these patients with consistent and co-ordinated information regarding their disease and how it, and associated treatments, could affect conception or pregnancy. Results from this study suggest physicians should

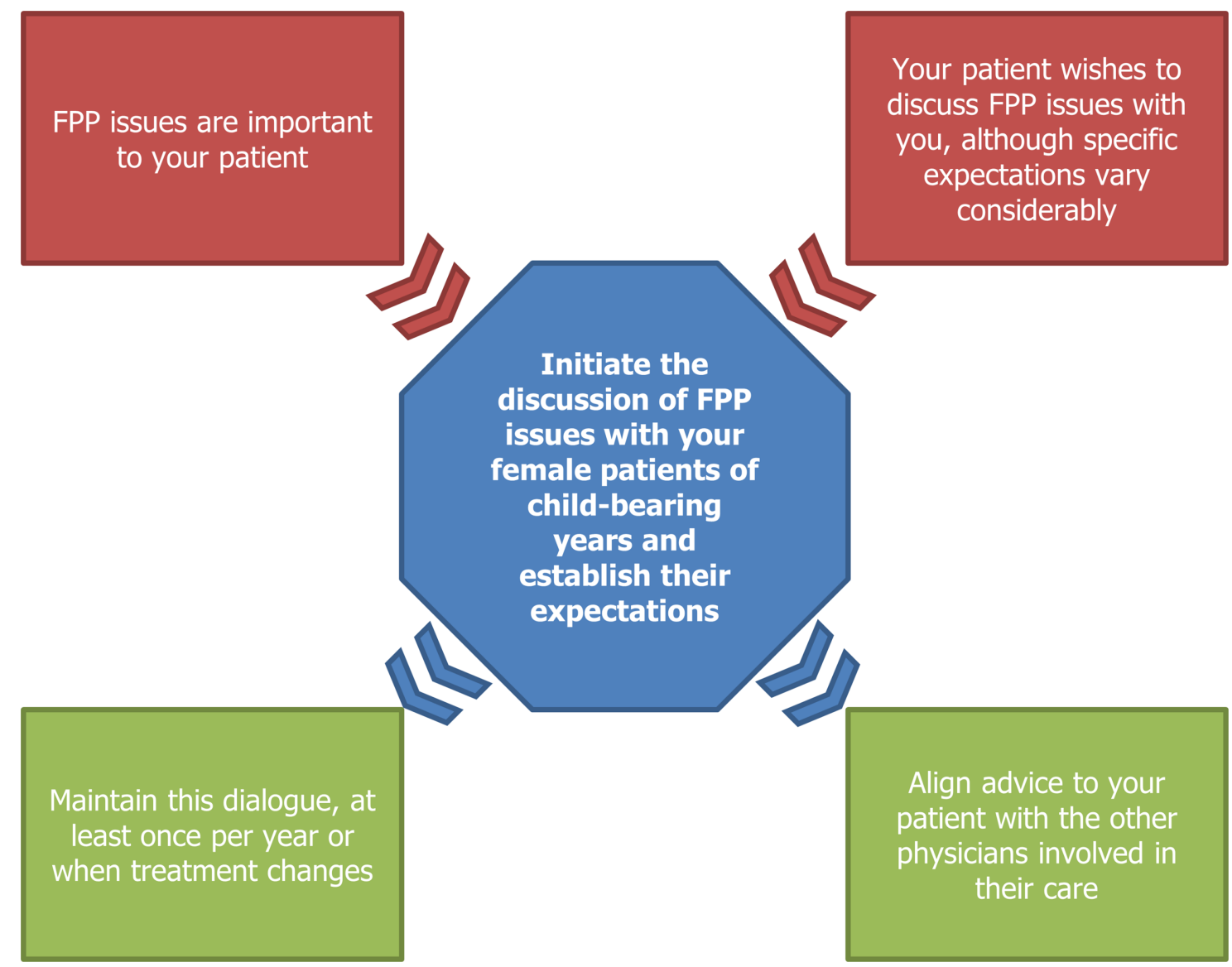

Figure 5 Family planning and pregnancy-related considerations for practicing physicians treating female patients of childbearing age who live with chronic inflammatory disease: key messages from the survey of current clinical practice and patient perceptions. 
regularly initiate discussion of these topics with their female patients of childbearing age, particularly those on chronic medication or when changes to the treatment plan could impact pregnancy outcome, in order to improve patient support. In addition, greater crossspecialty communication between physicians involved in different aspects of the patient's care, from gastroenterologists and rheumatologists to gynaecologists/obstetricians and GPs, is needed to improve the consistency of advice offered to this often overlooked patient population.

Acknowledgements This publication has been funded by UCB Pharma. The authors acknowledge InSites Consulting and ACROSS HEALTH for survey development, management and analysis. The authors also acknowledge Costello Medical Consulting for editorial and administrative support.

Contributors EC, MEBC, CG and DSP were involved in substantial contribution to conception and design, interpretation of the data, drafting and critical revision of the manuscript, and final approval of the manuscript for submission. SM was involved in substantial contribution to conception and design, acquisition, analysis and interpretation of the data, critical revision of the manuscript and final approval of the manuscript for submission.

Funding This work was supported by UCB Pharma.

Competing interests EC has previously been reimbursed by UCB Pharma for consultation services; MEBC has previously been reimbursed by UCB Pharma for consultation services; DSP is an employee of UCB Pharma; SM is an employee of UCB Pharma.

Provenance and peer review Not commissioned; externally peer reviewed.

Data sharing statement No additional data are available.

Open Access This is an Open Access article distributed in accordance with the Creative Commons Attribution Non Commercial (CC BY-NC 3.0) license, which permits others to distribute, remix, adapt, build upon this work noncommercially, and license their derivative works on different terms, provided the original work is properly cited and the use is non-commercial. See: http:// creativecommons.org/licenses/by-nc/3.0/

\section{REFERENCES}

1. Symmons D, Turner G, Webb R, et al. The prevalence of rheumatoid arthritis in the United Kingdom: new estimates for a new century. Rheumatology 2002;41:793-800.

2. Symmons DP. Epidemiology of rheumatoid arthritis: determinants of onset, persistence and outcome. Best Pract Res Clin Rheumatol 2002;16:707-22.

3. Carbone LD, Cooper C, Michet CJ, et al. Ankylosing spondylitis in Rochester, Minnesota, 1935-1989. Is the epidemiology changing? Arthritis Rheum 1992;35:1476-82.

4. Sieper J, Braun J. Ankylosing spondylitis in clinical practice. Springer-Verlag London Limited, 2011.

5. Binder V. Epidemiology of IBD during the twentieth century: an integrated view. Best Pract Res Clin Gastroenterol 2004;18:463-79.

6. Gollop JH, Phillips SF, Melton LJ, et al. Epidemiologic aspects of Crohn's disease: a population based study in Olmsted County, Minnesota, 1943-1982. Gut 1988;29:49-56.

7. Loftus EV Jr. Clinical epidemiology of inflammatory bowel disease: incidence, prevalence, and environmental influences. Gastroenterology 2004;126:1504-17.

8. Klippel JH. Systemic lupus erythematosus: demographics, prognosis, and outcome. J Rheumatol Suppl 1997;48:67-71.
9. Bush MC, Patel S, Lapinski RH, et al. Perinatal outcomes in inflammatory bowel disease. J Matern Fetal Neonatal Med 2004;15:237-41.

10. Morales M, Berney T, Jenny A, et al. Crohn's disease as a risk factor for the outcome of pregnancy. Hepatogastroenterology 2000:47:1595-8.

11. Ostensen M. Sex hormones and pregnancy in rheumatoid arthritis and systemic lupus erythematosus. Ann NY Acad Sci 1999;876:131-43; discussion 44.

12. Baird DD, Narendranathan M, Sandler RS. Increased risk of preterm birth for women with inflammatory bowel disease. Gastroenterology 1990;99:987-94.

13. van Dunne FM, Lard LR, Rook D, et al. Miscarriage but not fecundity is associated with progression of joint destruction in rheumatoid arthritis. Ann Rheum Dis 2004;63:956-60.

14. Jawaheer D, Zhu JL, Nohr EA, et al. Time to pregnancy among women with rheumatoid arthritis. Arthritis Rheum 2011;63:1517-21.

15. Mayberry JF, Weterman IT. European survey of fertility and pregnancy in women with Crohn's disease: a case control study by European collaborative group. Gut 1986;27:821-5.

16. Manosa M, Navarro-Llavat M, Marin L, et al. Fecundity, pregnancy outcomes, and breastfeeding in patients with inflammatory bowel disease: a large cohort survey. Scand J Gastroenterol 2013;48:427-32.

17. Vermeire S, Carbonnel F, Coulie PG, et al. Management of inflammatory bowel disease in pregnancy. $J$ Crohns Colitis 2012;6:811-23.

18. Mahadevan U, Cucchiara S, Hyams JS, et al. The London Position Statement of the World Congress of Gastroenterology on Biological Therapy for IBD with the European Crohn's and Colitis Organisation: pregnancy and pediatrics. Am J Gastroenterol 2011;106:214-23; quiz 24.

19. Ng SW, Mahadevan U. Management of inflammatory bowel disease in pregnancy. Expert Rev Clin Immunol 2013;9:161-74.

20. Ostensen M, Forger F. Management of RA medications in pregnant patients. Nature Rev Rheumatol 2009;5:382-90.

21. Syme MR, Paxton JW, Keelan JA. Drug transfer and metabolism by the human placenta. Clin Pharmacokinet 2004;43:487-514.

22. Buckley LM, Bullaboy CA, Leichtman L, et al. Multiple congenital anomalies associated with weekly low-dose methotrexate treatment of the mother. Arthritis Rheum 1997:40:971-3.

23. Lloyd ME, Carr M, McElhatton P, et al. The effects of methotrexate on pregnancy, fertility and lactation. QJM 1999;92:551-63.

24. Brent RL. Teratogen update: reproductive risks of leflunomide (Arava); a pyrimidine synthesis inhibitor: counseling women taking leflunomide before or during pregnancy and men taking leflunomide who are contemplating fathering a child. Teratology 2001;63:106-12.

25. Chakravarty EF, Sanchez-Yamamoto D, Bush TM. The use of disease modifying antirheumatic drugs in women with rheumatoid arthritis of childbearing age: a survey of practice patterns and pregnancy outcomes. J Rheumatol 2003;30:241-6.

26. Langen ES, Chakravarty EF, Liaquat $\mathrm{M}$, et al. High rate of preterm birth in pregnancies complicated by rheumatoid arthritis. Am J Perinatol 2013;31:9-14.

27. Hameen-Anttila K, Jyrkka J, Enlund $\mathrm{H}$, et al. Medicines information needs during pregnancy: a multinational comparison. BMJ open 2013;3:e002594.

28. Peters SL, Lind JN, Humphrey JR, et al. Safe lists for medications in pregnancy: inadequate evidence base and inconsistent guidance from Web-based information, 2011. Pharmacoepidemiol Drug Saf 2013;22:324-8

29. Toomey D, Waldron B. Family planning and inflammatory bowel disease: the patient and the practitioner. Fam Pract 2013;30:64-8.

30. van der Woude CJ, Kolacek S, Dotan I, et al. European evidenced-based consensus on reproduction in inflammatory bowel disease. J Crohns Colitis 2010;4:493-510.

31. Finer LB, Henshaw SK. Disparities in rates of unintended pregnancy in the United States, 1994 and 2001. Perspect Sex Reprod Health 2006;38:90-6.

32. Panchal S, Khare M, Moorthy A, et al. Catch me if you can: a national survey of rheumatologists and obstetricians on the use of DMARDs during pregnancy. Rheumatol Int 2013;33:347-53. 